\title{
Penetrating brain injury
}

\author{
Sharma AP \\ Department of Surgery, Lumbini Medical College and Teaching Hospital, Tansen-11, Pravas, Palpa, Lumbini Zone, Nepal \\ Corresponding author: Prof. Achyut Prasad Sharma, FRCS(Ed), Neurosurgeon and Head Department of Surgery, Lumbini \\ Medical College and Teaching Hospital, Tansen-11, Pravas, Lumbini Zone, Nepal; e-mail: achyut-s@hotmail.com
}

\section{ABSTRACT}

In the past 20 years, there has been an increase in the incidence of head injuries caused by gunshot wounds. Penetrating brain injury is a traumatic brain injury caused by high-velocity projectiles or low-velocity sharp objects. A wound in which the projectile breaches the cranium but does not exit is referred as a penetrating wound, and an injury in which the projectile passes entirely through the head, leaving both entrance and exit wounds, is referred to as a perforating wound. A large number of these patients who survive their initial wounding will nevertheless expire shortly after admission to the hospital. Until the introduction of aseptic surgery in the last quarter of the nineteenth century, penetrating missile injuries of the brain were almost universally fatal. We have learned a great deal about gunshot wounds and their management from military experience gained during times of war, when a large number of firearm-related casualties are treated in a short period of time. ${ }^{1}$ Newly designed protective body armor has reduced the incidence of penetrating brain injuries significantly. Many of the victims in the vicinity of a cased explosive or an improvised explosive device will incur injuries by fragments. Blast injury is a common mechanism of traumatic brain injury among soldiers serving in war zone. Each war has had different lessons to teach. World War I for example, proved the efficacy of vigorous surgical intervention. During World War II, the importance of initial dural repair and antibiotic medication was first, debated, then acknowledged, and finally, universally accepted. The incidence of blast-induced traumatic brain injury has increased substantially in recent military conflicts. Blast-induced neurotrauma is the term given to describe an injury to the brain that occurs after exposure to a blast. Resent conflict has exposed military personnel to sophisticated explosive devices generating blast overpressure that results in secondary cellular and molecular insults to the brain parenchyma akin to diffuse brain injury. In soldiers with varying amounts of body armor, the pattern is quite different. What had previously been fatal penetrating brain injuries now become treatable brain injuries as a consequence of secondary damping of energy by the helmet. Traumatic brain injury is not prevented by a protective helmet. High- and low-frequency blast waves disrupt the blood-brain barrier and produce massive brain swelling in a very short time, thereby necessitating urgent decompressive craniectomy, and when low in energy, such blast waves may result in cytoskeletal and diffuse axonal injury that leads to neurodegeneration. Penetrating traumatic brain injury is typically identified and treated immediately mild traumatic brain injury may be missed, particularly in the presence of other more obvious injuries. In recent years there has been an apparent paradigm shift of scientific interest in long-term effects of mild traumatic brain injury and its contribution to posttraumatic stress disorder. ${ }^{1,14}$ The introduction of Guidelines for the Management of Penetrating Brain Injury has revolutionized the medical and surgical management of penetrating brain injury during the last decade 1 . There has been a paradigm shift toward a less aggressive debridement of deep seated fragments and a more aggressive antibiotics prophylaxis in an effort to improve outcomes.

Keywords: Penetrating brain injury, debridement, less aggressive surgical management.

\section{INTRODUCTION}

Gunshot wounds are a major health problem. Many deaths are due to gunshot wounds to the head, which are the most lethal. In the spring of 1998, the International Brain Injury Association, the Brain Injury Association, USA, the American Association of Neurological Surgeons, and the congress of Neurological Surgeons began work on the formulation of standard medical and surgical management for penetrating brain injury patients. Thus, Guidelines for the Management of Penetrating Brain Injury was published in 2001, which attempted to standardize both the medical and surgical management of penetrating craniocerebral trauma. Optimum management of penetrating brain injury requires a good understanding of ballistic characteristics of the wounding agents and the mechanism of wounding and the tissue damage and adherence to basic surgical principles are prerequisites to a carefully executed and definitive surgical management, when indicated'.

\section{BALLISTICS AND PATHOLOGY}

A firearm is any weapon that uses an explosive powder to propel a projectile. Firearms are classified based on their size, their muzzle velocity and type of projectile fired. The ability of bullets, shrapnel, and low velocity 


\section{Journal of Lumbini Medical College}

objects such as knives and arrow to penetrate the brain is dependent on their energy, shape, the angle of approach, and characteristics of intervening tissues. Primary injury to the brain is determined by the ballistic properties. There are three components to ballistics: interior, exterior and terminal. The behavior of a projectile on impacting its target is terminal ballistics. Interior ballistics is defined as the science of motion of a projectile through a gun barrel as a result of combustion and expansion of a powder charge. Exterior ballistics is the projectile's behavior in a medium such as air and is dependent on the projectile's shape, caliber, weight, initial velocity, and ballistic coefficient. Most handguns and revolvers use heavy bullets and have muzzle velocities ranging from 550 to $900 \mathrm{ft} / \mathrm{sec}$. These are referred to as low velocity missiles. In contrast, most of today's rifles use very light bullets and have muzzle velocities averaging $3000 \mathrm{ft} / \mathrm{sec}$, with a range of 2300 to $6000 \mathrm{ft} / \mathrm{sec}$. The wounding energy $(\mathrm{E})$ of a projectile depends mainly on its weight and velocity; hence, $\mathrm{E}=$ $1 / 2 M\left(V I^{2}-V R^{2}\right)$, where $M$ is the projectile's weight, $V I$ is the impact velocity, and VR is the residual velocity if the projectile has a perforating mode. Translation of kinetic energy into tissue damage is brought about by the tremendous amount of crushing pressure exerted on the brain parenchyma. Juxtamissile pressure affects the brain tissue immediately in the path of a projectile and may be in the thousands of atmospheres. Longitudinal strong shock waves start immediately after impact of the projectile with brain tissue and travel in spheres ahead of the projectile with velocities in excess of $1460 \mathrm{~m} / \mathrm{sec}$. Shock waves last up to $10 \mu \mathrm{sec}$ and measure up to $80 \mathrm{~atm}$. Ordinary pressure waves measuring up to 20 to $30 \mathrm{~atm}$ are generated as the projectile transfers its kinetic energy to the surrounding brain tissue and produces a temporary cavity. The negative pressure generated by the temporary cavity can suck contaminated material into the cavity. Every cycle of temporary expansion and collapse creates significant surrounding tissue injury to the brain. ${ }^{6,11}$ This can result in shear-like injury of the neurons or can result in epidural hematomas, subdural hematomas, or parenchymal contusions. The low-velocity sharp objects, which lack any definable ballistics, penetrate the scalp, skull, and dura and lacerate the brain parenchyma, including the cortex, subcortical white matter, basal ganglia, and diencephalon or brain stem and any blood vessels in their path. Similarly, low-velocity projectiles from artillery shells, improvised explosive devices, and spent bullets cut into the brain just like sharp objects do. Fragments of high-explosive devices are of various shapes and sizes and can weigh as much as $100 \mathrm{~g}$. These should be regarded as high-velocity missiles, because initially they travel at speed of over $3000 \mathrm{ft} / \mathrm{sec}$, although they rapidly lose speed because of their volume, irregular shape, and aerodynamic instability and become low-velocity missiles at distances as near as 10 meters.
The extent of tissue damage depends on the amount of energy expended by the missile at the point of tissue penetration. As the projectile travels through the brain parenchyma, it is preceded by transient sonic wave $(2 \mu \mathrm{s})$ which appears to have minimal influence on surrounding tissue. The projectile itself, however, crushes the soft brain tissue in its path, creating a permanent track of injury. This is in addition to the secondary missiles such as bone and metal fragments created from the impact of projectile on the skull. Additionally, a penetrating injury is expected to be much more severe in case of a close range firearm injury as maximum amount of initial kinetic energy is transferred to the brain tissue.

\section{CLINICAL FINDINGS}

Most patients involved in civilian gunshot wounds to head are male $(87 \%)$ in the third to fourth decade of life and are nearly equally divided between homicides (50\%) and suicides (46\%), with a small percentage being due to accidents (4\%). Military victims of penetrating brain injury tend to be younger. In civilian penetrating brain injury an altered level of consciousness is the rule. Glasgow Coma Scale score of the patients is used to assess the level of consciousness. ${ }^{7}$ When sharp objects, low-velocity and spent bullets penetrate the brain, they may cause focal deficits; however, if they do not disrupt the neuronal circuitry in the brainstem tegmentum or ascending reticular activating system, they may not cause a depressed level of consciousness.

\section{MANAGEMENT}

The Guidelines for the Management of Penetrating Brain Injury was adopted and published in The Journal of Trauma in August 2001, which has standardized both the medical and surgical management of these unique and challenging injuries. ${ }^{1}$ The pre-hospital rescue, intubation, oxygenation, ventilation, volume resuscitation, and medical management of patients with penetrating brain injury must clearly be adopted from different pre-hospital emergency department, critical care, and surgical guidelines. Immediately after arrival of the patient in the emergency department, a primary survey and stabilization of the patient with regard to the airway, breathing, cervical spine, and circulation including external hemorrhage should be achieved. After resuscitation, an inspection of superficial wound should be done. The skin, especially the scalp, must be examined meticulously for wounds as it may be covered by blood-matted hair. An entrance wound should be identified and its location recorded as well as any exit wounds when they exist. The superficial scalp should also be observed for powder burn, which would imply a close range firearm injury. Any cerebrospinal fluid, bleeding, or brain parenchyma oozing from the wound should be noted, the size of the deficit should also be documented. All orifices 


\section{AP Sharma}

must be checked for retained foreign bodies, the missile, teeth, and bone. A detailed neurological examination should be performed, and post-resuscitative Glasgow Coma Scale of the patients should be documented. A complete head to toe examination is recommended as penetrating brain injury patients may have multiple organ injuries. A detailed medical history from family or friends and a chronology of the incidence from a witness is warranted. Initial laboratory investigation must include a complete blood count, electrolytes, coagulation profile, blood grouping and crossmatching and blood gas analysis. Once the initial evaluation is done, the patient should have imaging studies. The utility of various neuroimaging methods used in patients with penetrating brain injury lies on the potential management and prognostic implications of these modalities. Plain radiographs of the skull can be considerable value in identifying the cranial wounds, the location of missile and bone fragments, and the presence of intracranial air. Computed tomography scanning of the head is now the primary modality used in the neuroradiologic evaluation of patients with penetrating brain injury. ${ }^{2}$ Computed tomography, including three-dimensional reconstruction of the head, defines the entry site and trajectory of the fragment into the brain, perforating, penetrating, or tangential terminal ballistics, and involvement of the paranasal sinuses, orbits, skull base, and mastoids. It defines the missile track, number of tracks and ricochet, whether the penetration is across the midsagittal or midcoronal planes, and the presence or absence of intracranial hematomas such as acute epidural, subdural, intracerebral, or intraventricular hematomas. The extent of brain edema and ischemia and brainstem involvement is defined by computed tomography. If a vascular injury is suspected, then cerebral angiography is recommended. The sensitivity to diagnose vascular injury such as traumatic dissection of the carotid or vertebral arteries with computed tomography angiography has been reported to be similar or even superior to that of magnetic resonance imaging angiography. In terms of other vascular pathology, the incidence of vasospasm in the setting of blast-related penetrating traumatic brain injury is high, approaching $50 \%$. Thus, it is recommended that patients with acute penetrating traumatic brain injury from explosives undergo regular noninvasive vascular assessment via transcranial Doppler, with follow-up invasive digital subtraction angiography for definitive diagnosis and endovascular intervention. Magnetic Resonance Imaging is generally not recommended but can be useful in penetrating brain injury caused by a wooden object. When stabilization and imaging are complete, decisions concerning further therapy often take into account the patient's neurologic status as determined by the Glasgow Coma Scale score. Poor survival and outcome are reported in patients with GCS scores between 3 and 5 points. Most neurosurgeons agree that a patient with a postresuscitation GCS score of 3 points with two dilated nonreactive pupils but without a mass lesion on $\mathrm{CT}$ should not receive surgical intervention. It has long been known that bihemispheric and transventricular injuries have poor prognosis.

\section{SURGICAL MANAGEMENT}

The general guidelines of surgical treatment include: adequate debridement of devitalized tissue, removal of the mass lesions, removal of the accessible in-driven bone fragments and foreign bodies, adequate haemostasis, dural reconstruction and complete closure of the scalp. The "Infection in Neurosurgery" Working Party of British Society for Antimicrobial Therapy recommended the following regimen for penetrating brain injury: intravenous co-amoxiclav $1.2 \mathrm{~g} \mathrm{q} 8 \mathrm{~h}$, or intravenous cefuroxime $1.5 \mathrm{~g}$, then $750 \mathrm{mg} \mathrm{q} 8 \mathrm{~h}$, with intravenous metronidazole $500 \mathrm{mg}$ $\mathrm{q} 8 \mathrm{~h}$. It is recommended that this regimen should be started as soon as possible after injury and continued for 5 days postoperatively. Scalp lacerations from missile head wounds are usually contaminated, have devitalized edges, and may be hard to repair. Treatment of small entrance wounds with local wound care and closure in patients whose scalp has not been devitalized and have no significant intracranial pathological findings on CT scan is not only adequate but recommended according to guidelines. When scalp is penetrated by a projectile, it is shredded, torn, or burned with devitalized edges of up to few millimeters, if feasible, it is strongly recommended that a plastic surgeon be consulted for primary closure of the skin over a torn dura, especially if the dural tear is at the base in the vicinity of the basal cisterns, near the air sinuses or mastoid air cells, to prevent CSF fistulas. Once a patient has been classified as a surgical candidate, attempts should be made to operate within 12 hours of injury to prevent infection and resulting abscesses. ${ }^{7}$ In the presence of significant mass effect, debridement of necrotic brain tissue along with safely accessible bone fragments is recommended. Brandvold and colleagues, Taha and associates, and more recently Amirjamshidi and coworkers, based on their experience in Israel, Lebanon, and Iran, respectively, have reported on minimal debridement of missile head wounds in special circumstances. ${ }^{2}$ Similarly, during arm-conflict in Nepal, the penetrating brain injuries were managed by less aggressive surgical debridement of devitalized brain tissue, removal of easily accessible metal and in-driven bone fragments with watertight closure of the dura mater at Birendra Hospital. ${ }^{10}$ It should be noted that any deeply seated bone fragments especially those in eloquent brain areas should not be retrieved because this has been shown to correlate with worse outcomes. This has marked a significant trend since Vietnam era to proceed with a more conservative, minimally invasive approach toward cerebral debridement as this has been shown to improve outcomes and lower morbidity. As with bone fragments, only accessible missile fragments in non- 


\section{Journal of Lumbini Medical College}

eloquent brain should be retrieved although there has been some suggestion that removal of all missile fragments may decrease the risk of seizures. Intracranial haematomas with significant mass effects should be evacuated. Although craniectomies around the entrance site of a projectile have been the favored technique in previous military conflicts, the present recommendation is craniotomy and debridement of the skull with replacement of the bone to avoid the future need for cranioplasty. Recent wartime explosive injuries from a transfrontal or transtemporal direction may disrupt the anterior and lateral skull base. Such injuries are associated with significant risk for CSF leaks and loss of anatomic continuity between the anterior cranial fossa, orbits, maxilla, and infratemporal fossa. During the recent conflict this has led to an aggressive strategy of early skull base repair with titanium mesh, local pericranium, fat, temporalis fascia, and muscle.

\section{COMPLICATIONS}

Patients who survive penetrating craniocerebral injuries are at risk of experiencing multiple complications, including persistence neurological deficits, infections, epilepsy, CSF leak, cranial nerve deficits, pseudoaneurysms, arteriovenous fistulas, and hydrocephalus. The principal objectives when treating patients with craniocerebral missile wounds are to lower morbidity and mortality and begin rehabilitation. Extensive studies have evaluated long-term survival and cognitive, behavioral, and functional outcome after PBI, especially in war injuries sustained in World War II and Korean and Vietnam conflicts. ${ }^{5,13}$ For patients who survive a $\mathrm{PBI}$, the size and location of the parenchymal injury may have a long-lasting effect on intelligence test scores. The focal motor deficits with or without sensory deficit can be hemiparesis, monoparesis, triparesis, paraparesis, pseudobulbar palsy, ataxia or spastic ataxia, quadriparesis, visual field deficit, cranial nerve deficit, speech difficulty etc. Depending on the entrance site, a persistent focal neurological deficit is quite common in the survivors. The incidence of motor deficits, visual field cuts, and speech difficulties in the casualties from the Iran-Iraq War was $34.6 \%, 13.7 \%$, and $6.1 \%$, respectively.

\section{INFECTIONS}

Since the early 1900s, various factors have been blamed for the high rate of infection in war wounds like delays in evacuation of casualties, inadequate debridement and inability to close dura and skin watertight, lack of antibiotics, coma, extent of injuries, especially multiple lobe and ventricle involvement, retained bone fragments and cerebrospinal fluid fistulas. Missile head wounds are contaminated wounds. The extent to which contaminating organisms contribute to deep wound infections is debatable. Deep infection remains the most important aspect of $\mathrm{PBI}$ that a neurosurgeon has to deal with. A follow-up revealed that in a population of 1221 patients with penetrating cerebral trauma incurred in the Vietnam War, there was 3 percent incidence of brain abscess. ${ }^{4}$ This complication usually occurred during the second or third week of injury. No relationship existed between the presence of retained fragments and the development of either a seizure disorder or a subsequent CNS infection. Overall, past military experiences have shown that timely evacuation and prophylactic institution of broad-spectrum antibiotics followed by careful debridement of penetrating craniocerebral wounds significantly reduce the incidence of CNS infection. CSF fistulas should be treated most expeditiously to prevent the severe neurological sequelae of the CNS infection. Dehiscence of a scalp flap incision is usually a result of infection in an otherwise healthy young trauma victim, but it can result from a failure in technical aspect of dural or scalp closure.

\section{POSTTRAUMATIC EPILEPSY}

The relation of epilepsy to brain trauma has been recognized since the days of Hippocrates, but the pathogenesis of the posttraumatic epilepsy is still not clearly understood. Patients with focal neurological signs or large lesions has increased risk of epilepsy, and the site of the lesion may have been more important than size in determining in occurrence. Traumatic brain injury greatly increases the risk for a number of mental health problems and is one of the most common causes of medically intractable epilepsy in humans6. Several models of traumatic brain injury have been developed to investigate the relationship between trauma, seizures, and epilepsy-related changes in neural circuit function. These studies have shown that the brain initiates immediate neuronal and glial responses following an injury, usually leading to significant cell loss in areas of the injured brain. Over time, long-term changes in the organization of neural circuits, particularly in neocortex and hippocampus lead to an imbalance between excitatory and inhibitory neurotransmission and increased risk for spontaneous seizures. These include alterations to inhibitory interneurons and formation of new, excessive recurrent excitatory synaptic connectivity. ${ }^{6}$ Penetrating brain injury is one of the major risk factor for posttraumatic epilepsy. Studies of veterans from World War I, World War II, and the Korean, Vietnam, and Iran-Iraq wars indicate that between $34 \%$ and $50 \%$ victims of penetrating brain injury become epileptic when monitored for 2 to 15 years. Follow-up studies indicated that the incidence of posttraumatic epilepsy in victims of $\mathrm{PBI}$ is higher than that in victims of closed head injury. ${ }^{5}$ The guidelines for "Management and prognosis of Penetrating Brain Injury" recommend prophylactic anti-seizure medications for the first week after PBI but not beyond that.

\section{CEREBROSPINAL FLUID LEAK}




\section{AP Sharma}

Cerebrospinal fluid fistulas in penetrating craniocerebral wounds constitute a serious complication, which can increase morbidity and mortality. Cerebrospinal fluid leak develops because of the dural tear by the missile along with a failure to adequately seal the defect by normal tissue healing processes. ${ }^{8}$ CSF leaks can present through the entry or exit sites of the projectile as well as through the ear or nose when the mastoid hair cells and open air sinuses have been violated, respectively. The drainage of CSF from the site of operative debridement is often caused by incomplete closure of the dural laceration at the base of the skull or by a convexity dural suture line that is too tight. If the CSF leak develops several days later, in the absence of hydrocephalus or of mass effect and/or haematoma, one should assume and treat for wound infection. When there is an injury of the frontal fossa floor, recurring CSF leaks usually result from an initial incomplete exploration of the fossa floor. During the recent conflict this has led to an aggressive strategy of early skull base repair with titanium mesh, local pericranium, fat, temporalis fascia, and muscle. This step is followed by more extensive skull base reconstruction at level five medical centers consisting of rotational or myocutaneous free flaps in patients with persistent CSF leaks, progressive skull base deformity, and encephalocele.

\section{PSEUDOANEURYSMS AND ARTERIOVENOUS FISTULAS}

Vascular injuries are thought to be one of the main causes of field mortality among patients with missile wounds to the head. Traumatic intracranial aneurysms are relatively rare lesions most commonly associated with penetrating wounds of the brain. ${ }^{9}$ Traumatic aneurysms are formed after the partial or complete rupture of the arterial wall. The true incidence of traumatic neurovascular injury in patients with blast overpressure, closed brain injury, and $\mathrm{PBI}$ remains largely speculative. ${ }^{12}$ Although first described in 1895 by Guibert, traumatic intracranial aneurysms have received little attention in the literature. The incidence of traumatic internal carotid artery aneurysm (TICA) after such injuries ranges from $3 \%$ to $40 \%$, depending on the timing of imaging studies. The timing of angiography after a missile head wound may be an important factor in the detecting aneurysms. In one report from the Iran-Iraq War, cerebral angiography an average of 17 days after missile head wounds in 255 patients disclosed 8 aneurysms (3\%). Jinkins and coworkers performed cerebral angiography within 24 hours of gunshot wounds to the head in 12 patients and found 3 internal carotid/vertebral and one combined aneurysm/arteriovenous fistula (33\%). Risk factors include orbitofaciocraniocerebral injuries, injuries near the pterion, and patients harboring intracranial haematomas. It is recommended that any patient with these risk factors undergo either $\mathrm{CT}$ angiography or conventional angiography to rule out TICA. These injuries are treated endovascularly with either coiling or stentassisted coiling, which resulted in preservation of the parent artery. Despite endovascular treatment some patients need definitive clip exclusion. Before the first reported series of endovascular management of TICAs in 1993, treatment options available to the neurosurgeons were limited to balloon occlusion, balloon trapping, copper wire thrombosis, surgical ligation, and clipping or occlusion of the parent artery with or without bypass. Endovascular techniques are the methods of choice for the treatment of patients with carotid cavernous fistulas. There is no general consensus regarding optimal management of internal carotid artery dissection, but the choice among medical, endovascular, and surgical options may depend on the type of injury, the anatomic location, the mechanism of injury, coexisting injuries, and comorbid conditions. Anticoagulant therapy should be initiated when a thrombus is detected.

\section{OUTCOME AND PROGNOSIS}

Penetrating brain injury, though less prevalent than closed head trauma, carries a worse prognosis. Many studies have attempted to associate various prognostic factors with outcome. The most important prognostic factor currently recognized is the Glasgow Coma Scale after cardiopulmonary resuscitation. Traditionally, the higher the GCS after resuscitation, the better the patient outcome. Extensive studies have evaluated long-term survival and cognitive, behavioral, and functional outcome after PBI, especially in war injuries sustained in World War II and Korean and Vietnam conflicts. The Vietnam Head Injury Study has demonstrated that there are no truly silent area of the brain when it comes to penetrating injuries. Regardless of how well patients seem to recover, very complex psychobehavioral and cognitive functions are adversely affected, and community adjustment is never perfect. For this reason, every approach to the treatment of penetrating head injury must emphasize the preservation of brain tissue. A critical factor in early treatment decisions and long-term outcome after penetrating head injuries is the patient's initial level of consciousness. ${ }^{13}$ Low Glasgow Coma Scale is associated with an unfavorable outcome in both civilian and military PBI. GCS score of 3 with bilaterally fixed and dilated pupils, and high initial intracranial pressure have been correlated with worse outcomes in PBI patients. Increasing age, suicide attempt, coagulopathy, bihemispheric lesion, multilobar injuries, intraventricular haemorrhage, subarachnoid haemorrhage, transventricular injury, uncal herniation, respiratory distress and hypotension all are associated with poor outcome. There is evidence that soldiers returning home from combat duties in Iraq and Afghanistan may suffer from poor general 


\section{Journal of Lumbini Medical College}

health and be more susceptible to cardiovascular complications, both of which can ultimately affect their job performance and productivity. It is possible that mild traumatic brain injury (MTBI) under unusually stressful circumstances, such as blast injuries resulting from the explosive effects of improvised explosive devices (IEDs), may affect the neuronal circuitry designed to monitor stressful conditions, such as the amygdala, lateral hypothalamus, and pituitary/adrenal axis. This effect may result in excessive amount of stressful hormones and icosanoids and deposition of implicit memory of trauma that will result in posttraumatic stress disorder. A multiphasic research project is currently ongoing to investigate pathogenesis and best ways to manage MTBI and PTSD. Research in this area is highly warranted as PBI patients still present a significant challenge to practicing neurosurgeons worldwide. Patients with craniocerebral missile wounds who arrive at the hospital alive often receive variable treatment despite low GCS scores and often dismal prognoses, because there is a lack of consensus regarding appropriate treatment and predictable outcome in these patients. In particular, wide variations exist in the amount of surgical debridement performed, the use of ICP monitoring, and the use of various medical therapies.

\section{CONCLUSION}

Gunshot wounds of the head are on increase. Penetrating head injuries can be the result of numerous intentional or unintentional events, including missile wounds, stab wounds, and motor vehicle or occupational accidents (nails, screwdrivers). The pathological consequences of penetrating head wounds depend on the circumstances of the injury, including the properties of the weapon or missile, the energy of the impact, and the location and characteristics of the intracranial trajectory. The clinical condition of the patient depends mainly on the mechanism, anatomical location of the lesions, and associated injuries. The assessment of patients with penetrating brain injuries should include routine laboratory tests, coagulation profile and imaging studies. Patients with severe penetrating brain injuries should receive resuscitation according to Advanced Trauma Life Support Guidelines. The introduction of Guidelines for the Management of Penetrating Brain Injury has revolutionized the medical and surgical management of PBI during the last decade. There has been a paradigm shift toward a less aggressive debridement of deep seated fragments and a more aggressive antibiotics prophylaxis in an effort to improve outcomes. ${ }^{11}$ Morbidity and mortality rates associated with penetrating brain injury remain unacceptably high. Considerable research continues in the area of neurotrauma. Once the secondary mechanisms of injury are better understood and the treatment modalities are studied in prospective randomized clinical trials, less variation in management of penetrating head injury is likely to occur. The medical community as a whole will become more successful in the treatment of these patients.

\section{REFERENCES}

1. Benny Brandvold, M.D., Lion Levi, M.D., Moshe Feinsod, M.D. et al. Penetrating craniocerebral injuries in the Israeli involvement in the Lebanese conflict, 1982-1985. Analysis of a less aggressive surgical approach. J Neurosurg 1990; 72: 15-21.

2. Bizhan Aarabi B, M.D., F.R.C.S.C. Surgical outcome in 435 patients who sustained missile head wounds during the Iran-Iraq War. J Neurosurg 1990; 27: 692-695.

3. Espocito, Domenic P. MD, FACS; Walker, James B. MD. Contemporary Management of Penetrating Brain Injury. Neurosurg Quarterly 2009; 19: 249-254.

4. Aarabi, Bizhan MD; Taghipour, Musa MD; Alibaii, Ehsanali MD. et al. Central nervous system infections after military missile head wounds. J Neurosurg 1998; 42: 500-9.

5. Andres M. Salazar, MD; Bahman Jabbari, MD; Stephen C vance, MD. et al. Epilepsy after penetrating head injury. I. Clinical correlates: A report of the Vietnam Head Injury Study. J Neurol 1995; 35: 1406-14.

6. Robert F. Hunt. Jeffery A. Boychuk. Bret N, Smith. Neural circuit mechanisms of posttraumatic epilepsy. Journal Frontiers in Cellular. Neuroscience 2013; 7: 89.

7. Syed Farak Kazim, Muhammad Shahzad Shamim Muhammad Zubair Tahir, Syed Ather Enam. et al. Management of penetrating brain injury. Journal of Emergencies, Trauma, and Shock. Jul-Sec; 4: PP 395- 402.

8. Arnold M. Meirowsky, MD; William F. Caveness, MD; James D. Dillon, MD. et al. Cerebrospinal fluid fistulas complicating missile wounds of the brain. J Neurosurg 1981; 54: 44-8.

9. Sharma A.P, MD, FRCS(Ed). Nature and management of penetrating craniocerebral missile injuries at Shree Birendra Hospital from November 2001 to November 2002. Med J Shree Birendra Hospital 2002; 5: 1-6.

10. Fuad Sami Haddad, MD, F.R.C.S.(C), F.A.C.S.; Georges F. Haddad, MD; and Jamal Taha, MD. Traumatic Intracranial Aneurysms Caused by Missiles: Their Presentation and Management. J Neurosurg 1991; 28: 1-7.

11. Michael E. Carey, M.D. The treatment of wartime brain wounds: Traditional versus minimal debridement. Surg Neurol 2003; 60: 112-9.

12. Bizhan Aaribi, M.D, F.R.C.S (C). Traumatic aneurysms of the brain due to high velocity missile head wounds. Journal of Neurosurgery 1998; 22: 1056-1063.

13. Michael L. Levy, M.D. Outcome prediction following penetrating craniocerebral injury in a civilian population: Aggressive surgical management in patients with admission Glasgow Coma Scale scores of 6 to 15 . Neurosurgery focus 1999; 8: Article 2.

14. Schneiderman Al, Braver ER, Kang HK. Understanding sequelae of injury mechanisms and mild traumatic brain injury incurred during the conflicts in Iraq and Afghanistan: Persistent post concussive symptoms and posttraumatic stress disorder. Am J Epidemiol 2008; 167: 1446-52. 